HEPATOLOGY

\title{
Atorvastatin protects obese mice against hepatic ischemia-reperfusion injury by Toll-like receptor-4 suppression and endothelial nitric oxide synthase activation
}

\author{
Hussam Ajamieh, ${ }^{*}$ Geoffrey Farrell, ${ }^{*}$ Heng Jian Wong, ${ }^{*}$ Jun Yu, ${ }^{\dagger}$ Eagle Chu, ${ }^{\dagger}$ Jeffrey Chen* and \\ Narci Teoh* \\ * Gastroenterology and Hepatology Unit, Australian National University Medical School, the Canberra Hospital, Australian Capital Territory, \\ Canberra, Australia; and 'Institute of Digestive Disease and Department of Medicine and Therapeutics, Li KaShing Institute of Health Sciences, \\ Hong Kong, China
}

\section{Key words}

atorvastatin, liver inflammation, nuclear factor- $\kappa B$, non-alcoholic fatty liver disease, Toll-like receptor-4.

\section{Accepted for publication 9 March 2012.}

\section{Correspondence}

Associate Professor Narci C. Teoh, Gastroenterology and Hepatology Unit, Level 2, Building 1, the Canberra Hospital, Yamba Drive, Garran, ACT 2605, Australia. Email: narci.teoh@anu.edu.au

\begin{abstract}
Background and Aim: Steatosis accentuates the severity of hepatic ischemia-reperfusion injury (IRI). 3-Hydroxy-3-methylglutaryl-coenzyme A reductase inhibitors ("statins") protect the heart and brain against post-ischemic injury, without necessarily lowering serum cholesterol. We tested whether 10-day or 1-day atorvastatin administration protects livers with fatty change or non-alcoholic steatohepatitis (NASH) against IRI.

Methods: Mice with dietary or genetic simple steatosis (SS) or NASH were subjected to $60 \mathrm{~min}$ of partial hepatic ischemia/24-h reperfusion, with/without atorvastatin administered with food $(5 \mathrm{mg} / \mathrm{kg}$ body weight) for 10 days, or injected intravenously $(5 \mathrm{mg} / \mathrm{kg}) 24 \mathrm{~h}$ before ischemia. Liver injury, Toll-like receptor-4 (TLR4), cytokines/chemokines, endothelial nitric oxide synthase (eNOS), activation and thromboxane B2 production were determined.

Results: Atorvastatin conferred $70-90 \%$ hepatic protection against IRI in obese animals with SS or NASH, in which IRI was accentuated twofold to fivefold. IRI markedly upregulated TLR4 and activated nuclear factor- $\kappa \mathrm{B}(\mathrm{NF}-\kappa \mathrm{B})$; atorvastatin abrogated these effects, as well as activating eNOS. Atorvastatin dampened the post-ischemic induction of thromboxane B2, macrophage inflammatory protein-1a, monocyte chemotactic protein-1, tumor necrosis factor- $\alpha$, interleukin (IL)-12 p40, $\gamma$-interferon, IL-6, and adhesion molecules (vascular cell adhesion molecule-1, E-selectin, vascular endothelial-cadherin), and reduced macrophage and neutrophil recruitment. There was no reduction in serum cholesterol that could explain these effects, and hepatic cholesterol was normal in these mice. A single 24-h injection of atorvastatin conferred equivalent hepatoprotection.

Conclusion: Statins exert major hepatoprotection against IRI in lean, fatty, and NASH livers that is not due to cholesterol removal. Rather, statins downregulate TLR4 to prevent $\mathrm{NF}-\kappa \mathrm{B}$ activation, with resultant suppression of adhesion molecules, chemokines/ cytokines, and thromboxane B2 production. Short-term statin treatment is an effective, readily-available preventive agent against hepatic IRI, irrespective of obesity and fatty liver disease.
\end{abstract}

\section{Introduction}

In liver transplantation, steatosis affecting at least $30 \%$ of hepatocytes might render donor organs more susceptible to ischemiareperfusion injury, leading to primary graft failure or accelerated rejection. Because of the high prevalence of non-alcoholic fatty liver disease (NAFLD), an unacceptable proportion of such "marginal" donor livers are discarded. ${ }^{1}$

The early events in IRI involve the sinusoidal endothelial cell (SEC), the key site of injury and cell death; ${ }^{2-4}$ it also plays a critical role in microcirculatory inflammatory recruitment during IRI. ${ }^{5}$ The later phase $(12-48 \mathrm{~h})$ is characterized by hepatic parenchymal inflammation with polymorphonuclear neutrophils (PMN) that target hepatocytes and SEC. Proposed mediators of this inflammatory response $\mathrm{e}^{4,6}$ include activation of nuclear factor- $\mathrm{\kappa B}(\mathrm{NF}-\kappa \mathrm{B})$, which induces the transcription of pro-inflammatory chemokines, cytokines, and adhesion molecules. NF- $\mathrm{KB}$ activation can be triggered by oxidative stress, ${ }^{7}$ activation of cell death receptors, or signaling via Toll-like receptor (TLR)-4. ${ }^{7,8}$ TLR4, which is expressed on Kupffer cells, SEC, and hepatocytes, is a key 
signaling molecule in IRI and non-alcoholic steatohepatitis (NASH). ${ }^{9,10}$

The susceptibility of fatty livers to IRI has been attributed to microvascular disturbance caused by fat-laden hepatocytes compressing into the sinusoidal lumen, ATP depletion, oxidative stress, and different inflammatory mediators than those found in the lean liver. ${ }^{1,11}$ We recently showed that the peroxisome proliferatoractivated receptor- $\alpha$ agonist, Wy-14,643, reduced the severity of hepatic IRI in fatty livers, including those with NASH. ${ }^{11}$ However, fibrates approved for human use are less potent than Wy-14,643.

In considering agents that confer properties of microvascular protection against post-ischemic injury, we noted that the vascular and tissue protective properties of 3-hydroxy-3-methylglutarylcoenzyme A reductase inhibitors, or "statins" have been demonstrated in many organs, including the myocardium ${ }^{12,13}$ and lean liver. ${ }^{14}$ These effects are not solely attributable to cholesterollowering properties, and include endothelial cell protection, antiinflammatory, antioxidant, and antithrombotic actions. ${ }^{15}$ These pleiomorphic effects are likely related to the proximal steps in cholesterol metabolism involving the formation of farnesyl and geranylgeranyl pyrophosphate precursors that activate regulatory proteins to promote their translocation from the cytosol to plasma membrane. Here, they engage such processes as endothelial cell survival, growth, migration, and inflammation. In addition, the inhibition of Ras and Rho isoprenylation by statins preserves the endothelial barrier by enhancing the concentration of nitric oxide via the induction and activation of endothelial nitric oxide (NO) synthase (eNOS). ${ }^{16}$

Others have recently reported that statins $(10 \mathrm{mg} / \mathrm{kg}$ gavaged for 7 days) protect against IRI in mice fed a $2 \%$ cholesterol high-fat diet (HFD), and that the mechanism of this effect was the lowering of mitochondrial cholesterol content by reducing hepatic cholesterol stores. ${ }^{17}$ However, this model is not related to the pathophysiology of human NAFLD, which involves overnutrition and insulin resistance, and which is only associated with hepatic cholesterol accumulation in the presence of NASH. ${ }^{18}$ We therefore tested the hypothesis that the oral administration of atorvastatin for 10 days would protect fatty livers caused by obesity and diabetes, including NASH, against hepatic IRI. By using animals known to have normal hepatic cholesterol and a regimen that does not involve gavaging or lowering of serum cholesterol, as well as testing a single and practical 24-h regimen, we demonstrate that the protective mechanisms are via anti-inflammatory (not cholesterollowering) effects, particularly the suppression of TLR4, which plays a key role in both IRI and NASH. We also show that statins directly influence microvascular function via the selective activation of eNOS.

\section{Methods}

Animal experiments and atorvastatin administration. The animal experiments were approved by the Australian National University (ANU) Animal Ethics Committee (Canberra, ACT, Australia). Except where otherwise indicated, we used male foz/foz (Alms1 mutant) mice to generate obesity-related fatty liver (NAFLD) or NASH, as genotypically and phenotypically characterized in earlier publications. ${ }^{19-22}$ Foz/foz mice and lean, wildtype (WT) littermates were housed at the ANU Medical School
Animal Facility under standard conditions. ${ }^{5,11}$ After weaning, the mice were fed normal chow (NC; 5.4\% w/w) or a HFD (23\% fat, $0.2 \%$ cholesterol w/w; SF03-020; Specialty Feeds, Glen Forrest, WA, Australia) for 8 weeks. Using two genotypes and either a HFD or NC feeding, we generated either lean mice (NC-fed WT), two different groups of obese mice with simple steatosis (SS) (HFD WT, or NC-fed foz/foz mice), and obese/diabetic mice with NASH (HFD foz/foz mice). Atorvastatin (Pfizer, Sydney, NSW, Australia), dissolved in $0.5 \%$ carboxymethyl cellulose, was mixed with diet $(0.2 \% \mathrm{w} / \mathrm{w})$ and estimated from daily food intake to approximate $5 \mathrm{mg} / \mathrm{kg}$ body weight/day. For intravenous use, atorvastatin (PZ0001; Sigma, St Louis, MO, USA) was prepared in DMSO and administered at a dose of $5 \mathrm{mg} / \mathrm{kg}$ body weight. Vehicle controls were injected with an equivalent volume of DMSO. Experimental procedures for $60 \mathrm{~min}$ hepatic IRI and sham controls are reported. ${ }^{11}$ In supplementary experiments, we subjected MyD88 ${ }^{-/-}$mice against hepatic IRI. After 24-h reperfusion, anesthetized mice were exsanguinated (cardiac puncture) for the preparation of plasma; the livers were harvested and stored at $-80^{\circ} \mathrm{C}(n=6$ per time-point/treatment group $) .{ }^{11}$

Assessment of liver injury. Serum alanine aminotransferase (ALT) was assayed by automated procedures (Clinical Pathology, The Canberra Hospital, ACT, Canberra, Australia). Necrotic areas in consecutive liver sections $(4 \mu \mathrm{m})$ were stained with hematoxylin-eosin (HE) and quantified using Image Pro 5.1 (Media Cybernetics, Silver Spring, MD, USA). ${ }^{11}$

Immunohistochemistry. The formalin-fixed, paraffinembedded liver was processed and quenched for endogenous peroxidase activity. Macrophages were stained using F4/80 (AbD Serotech, Oxford, UK) and PMN by myeloperoxidase (Abcam, Cambridge, UK).

\begin{abstract}
Measurement of serum chemokines and cytokines. An antibody-coupled, bead-based system (Bio-Plex mouse 23-plex assay; Bio-Rad, Hercules, CA, USA) was utilized to quantify serum levels of 23 chemokines and cytokines (detection to $32000 \mathrm{pg} / \mathrm{mL}$ ). Serum thromboxane B2 (TxB2) concentrations were measured by ELISA (Assay Designs, Ann Arbor, MI, USA).
\end{abstract}

Determination of hepatic mRNA species. We used reverse transcription, real-time polymerase chain reaction for the semiquantitation of genes of interest, as described. ${ }^{5,11}$ Relative mRNA expression eNOS was analyzed using iQ SYBR Green Supermix (Bio-Rad, USA) and Optical System Software version 2.0 (Bio-Rad, Hercules, CA, USA). The results were normalized to glyceraldehyde3-phosphate dehydrogenase mRNA.

Western blot analyses. After preparation of the hepatic lysates and nuclear extracts, ${ }^{5,11}$ protein $(30 \mu \mathrm{g})$ was resolved by 7.5-12\% sodium dodecylsulfate-polyacrylamide gel electrophoresis, transferred to polyvinylidene difluoride membranes (Millipore, Billerica MA, USA), and probed for phosphorylated eNOS (p-eNOS) $)^{\text {Ser1177 }}$ (Cell Signaling, Danvers, MA, USA), vascular cell adhesion molecule-1 (VCAM-1), HSP90 (R\&D System, 
Minneapolis, MN, USA), eNOS, inhibitor of $\kappa \mathrm{B}-\alpha$ (IкB- $\alpha$ ), p65, TLR4, E-selectin (Santa Cruz Biotechnology, Santa Cruz, CA, USA) and vascular endothelial-cadherin (BD Biosciences, Bedford, MA, USA). Densitometry was performed using Fujifilm

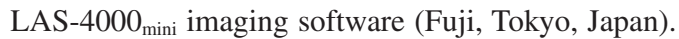

Statistical analyses. Non-parametric ANOVA with Tukey post-hoc analysis was used to compare data between either different groups of fatty liver pathology and lean liver, or the effects of IRI versus sham surgery, or for atorvastatin treatment versus no treatment or vehicle. Results (mean $+/-$ standard deviation) are considered significant when $P<0.05$.

\section{Results}

Oral atorvastatin for 10 days protects fatty and Iean livers against hepatic IRI. As reported previously ${ }^{11}$ and shown in Figure 1a, hepatic IRI was accentuated in this metabolic syndrome NASH model (HFD foz/foz), as well as in both dietary (HFD WT) and genetic (NC-fed foz/foz) groups, compared with lean mice (NC-fed WT). In both SS models, ALT values after IRI were increased approximately twofold above lean, and values were approximately fivefold higher in HFD foz/foz mice with NASH, compared with lean mice (Fig. 1a). Histological analysis confirmed more extensive necrotic areas after IRI in livers, showing NASH, than in both SS models, all 1.5-threefold greater than in the lean liver (Fig. 1b; Table 1). 10 days of oral atorvastatin failed to alter serum cholesterol in any group (Supplementary Table S1). Despite this, 10-day oral atorvastatin $(5 \mathrm{mg} / \mathrm{kg}$ body weight) reduced ALT levels after IRI by $70-90 \%$ in all livers, with $\sim 80 \%$ reduction in NASH (Fig. 1a). Likewise, these noncholesterol-lowering doses of atorvastatin afforded the robust preservation of hepatic architecture, with substantial reductions in the necrotic area (Fig. 1b; Table 1).

Oral atorvastatin treatment inhibits TLR4 expression and blocks NF-KB activation. NF- $\kappa B$ is activated by oxidative stress and inflammatory cytokines, and by TLR4, a key mediator of IRI. ${ }^{6,22-25}$ In the present experiments, IRI increased hepatic TLR4 expression at $24 \mathrm{~h}$ (Fig. 1c), and we confirmed independently that mice lacking MyD88, and an essential pathway linking TLR4 to NF- $\kappa \mathrm{B}$ activation, are refractory to IRI (Supplementary Fig. S1). In the two SS models, post-ischemia TLR4 expression was almost double that of the corresponding lean liver, while the TLR4 expression was amplified a further twofold in NASH (Fig. 1c). In all groups, IкB- $\alpha$ was significantly degraded at 24-h post-ischemic reperfusion (Fig. 1d), which was associated with increased nuclear p65 (Fig. 1e). Pretreatment with atorvastatin inhibited I $\mathrm{B} \mathrm{B} \alpha$ degradation and completely abrogated nuclear translocation of p65 at 24-h reperfusion. As for TLR4 blockade, this effect of atorvastatin on inhibiting NF- $\kappa \mathrm{B}$ activation was most pronounced in livers with steatosis or NASH (Fig. 1d,e).

Oral atorvastatin enhances expression and activation of eNOS. In several tissues (brain, kidney), statins exert protective effects against IRI by activating eNOS. ${ }^{15,26}$ In the present experiments, atorvastatin treatment, in the absence of IRI, increased hepatic eNOS mRNA (Fig. 2a). Because eNOS itself is an important surrogate marker of the efficacy of statins at the transcriptional level, total eNOS expression levels were quantified (Fig. 2b,c). eNOS protein expression did not appear to change in sham-operated mice (Fig. 2b); however, after IRI, the eNOS protein increased in all except one (dietary) steatosis group (Fig. 2c). Another indication of the role of eNOS for endothelial cell protection and microvascular perfusion is its activation state; atorvastatin treatment substantially increased the activity of eNOS after IRI, as reflected by the accumulation of the p-eNOS protein in mice with lean, simple steatotic and NASH livers (Fig. 2d).

\section{Oral atorvastatin treatment reduces post-} ischemic inflammatory responses in fatty and lean livers. In light of the above effects on NF- $\kappa \mathrm{B}$ activation, atorvastatin should exert anti-inflammatory effects after hepatic IRI related to the lower production of pro-inflammatory chemokines and cytokines. Serum macrophage inflammatory protein-1 (MIP-1a) (less consistently, monocyte chemotactic protein-1 [MCP-1]) increased approximately threefold after IRI in all groups; atorvastatin attenuated such MIP-1a induction in NASH and genetic SS, but not in dietary steatosis or lean mice (Table 2). IRI increased the synthesis and release of tumor necrosis factor- $\alpha$ (TNF- $\alpha$ ), interleukin (IL)-12 p40, $\gamma$-interferon (IFN- $\gamma$ ), and IL-6 (Table 2), and atorvastatin treatment exerted modest inhibitory effects on synthesis and release (by serum levels) of these cytokines after IRI in NASH, fatty, and lean livers (Table 2). Atorvastatin also suppressed the expression of E-selectin after IRI, irrespective of severity of fatty liver disease (Fig. 3a,b). Conversely, in the fatty liver groups, atorvastatin appeared to preserve expression of the endothelial cell marker vascular endothelialcadherin (Fig. 3c), which was otherwise suppressed after IRI, particularly in livers showing NASH. In all groups, IRI significantly upregulated VCAM-1 (Fig. 3d,e), and atorvastatin attenuated such VCAM-1 upregulation by approximately $50 \%$. Finally, atorvastatin substantially reduced TxB2 production in lean and fatty livers after IRI (Fig. 3f), although the similar trend in the NASH group was not statistically significant.

The late phase of hepatic IRI is characterized by parenchymal infiltration with macrophages and PMN; the latter initially adhering to the above adhesion molecules. ${ }^{3,5}$ In the present work, IRI at $24 \mathrm{~h}$ was associated with abundant PMN accumulation (Supplementary Fig. S1a), and atorvastatin treatment virtually abrogated such recruitment. We also showed that atorvastatin reduced hepatic macrophage accumulation at 24-h reperfusion in all groups, particularly with fatty livers (Supplementary Fig. S1b).

\footnotetext{
Single-dose atorvastatin administered intravenously $24 \mathrm{~h}$ before an ischemic insult also protects against hepatic IRI. These comprehensive findings indicate that pharmacological doses of atorvastatin given orally confer impressive hepatoprotective effects against hepatic IRI in lean, steatotic, or NASH livers without necessarily protecting hepatocyte mitochondria by cholesterol-lowering effects, as shown by others. ${ }^{17}$ However, the need for a 10-day treatment period would limit the clinical application of this strategy, and this might explain why there are still no reports of its adoption. ${ }^{27} \mathrm{We}$ reasoned that since hepatoprotection by atorvastatin against IRI in
} 
(a)

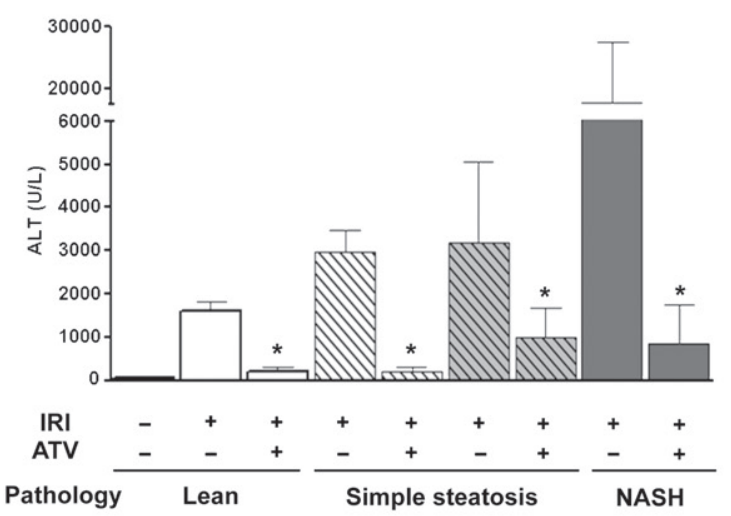

(b)

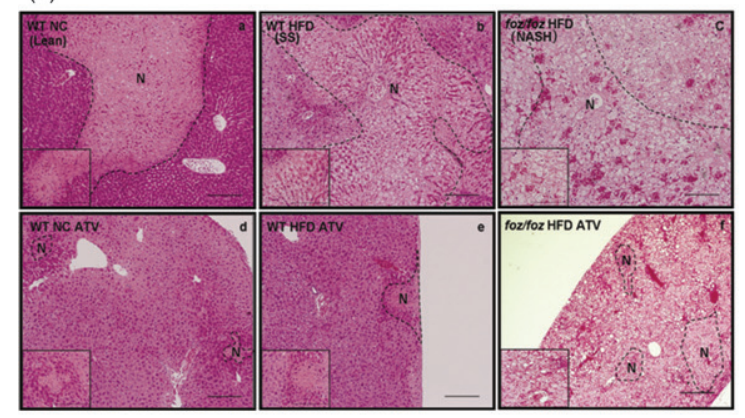

(c)

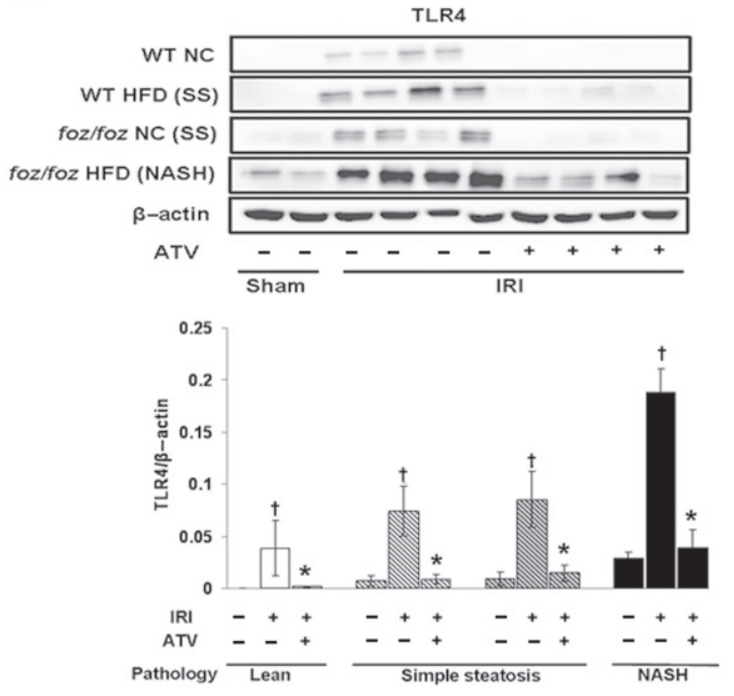

(d)
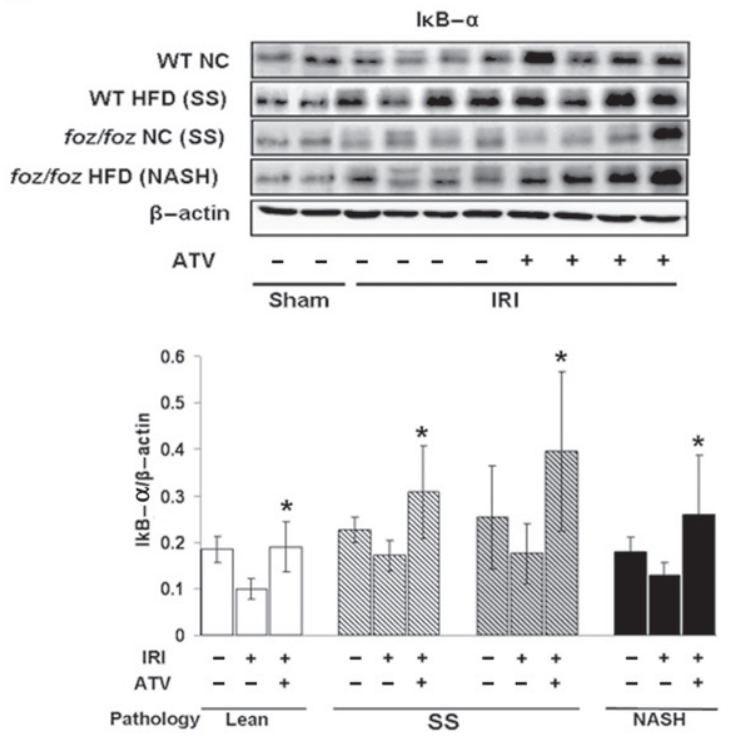

(e)

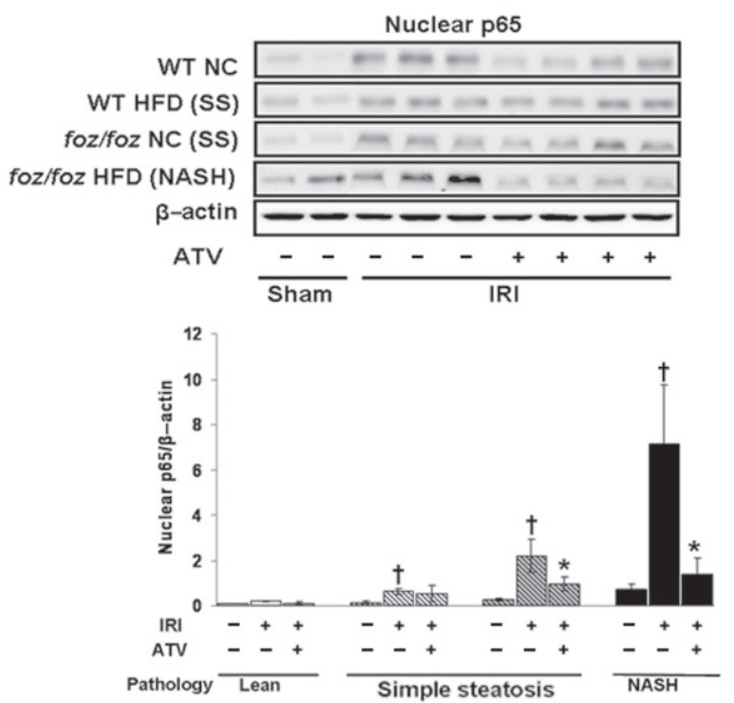

Figure 1 Effect of 10-day atorvastatin on serum alanine aminotransferase (ALT), liver histology, hepatic Toll-like receptor-4 (TLR4), inhibitor of $\kappa B-\alpha$ $(\mid \kappa B-\alpha)$, and nuclear p65 expression after $60 \mathrm{~min}$ of warm hepatic ischemia, followed by $24 \mathrm{~h}$ of reperfusion in non-alcoholic steatohepatitis (NASH), simple steatosis (SS), and lean livers. NASH was caused by feeding foz/foz mice a high-fat diet (HFD), SS either by feeding wild-type (WT) mice a HFD or foz/foz mice normal chow (NC) for 8 weeks. Lean mice were WT fed NC. (a) Serum alanine aminotransferase (ALT) levels. Data expressed as mean \pm standard deviation ( $n=6$ mice/group). ${ }^{*} P \leq 0.05$ compared to genotype-matched control with ischemia-reperfusion injury (IRI). Serum ALT levels in sham-operated atorvastatin-naïve animals were as follows: HFD foz/foz with NASH (93 \pm 6 U/L); NC-fed foz/foz mice with SS (63 \pm 15 U/L); WT mice fed HFD with SS (67 $\pm 15 \mathrm{U} / \mathrm{L})$; WT mice fed NC with lean liver (46 $\pm 15 \mathrm{U} / \mathrm{L})$. (b) hematoxylin-eosin (HE) staining shows extensive necrotic areas (N) for (a) lean, (b) SS (HFD WT mice), and (c) NASH (HFD foz/foz) mice. Atorvastatin-pretreated lean (d) and fatty liver (e) show near normal liver histology, and NASH (c) shows a substantial reduction in N. Representative HE of liver sections shown at high-power magnification (large panels: $\times 200$; inset panels: $\times 400$ ). (c) TLR4 protein, (d) IKB- $\alpha$, and (e) nuclear p65 shown in representative Western blots performed on hepatic lysates or nuclear extracts (for p65) from lean, steatotic (HFD WT mice) and NASH livers, as well as quantitation relative to $\beta$-actin. ${ }^{*} P \leq 0.05$ for effect of atorvastatin compared to genotype-matched controls with IRI. 
lean and fatty livers is related to the molecular suppression of TLR4, NF- $\kappa \mathrm{B}$, and the related post-ischemic inflammatory response, as well as the induction of eNOS, and not to cholesterollowering effects in our clinically-relevant models, such protection should not require more than a few hours of statin administration. We therefore repeated these experiments in mice with fatty livers, and administered atorvastatin $(5 \mathrm{mg} / \mathrm{kg})$ intravenously once only, $24 \mathrm{~h}$ before the hepatic ischemic insult. As indicated by serum

Table 1 Hepatic necrotic areas in mice after 60-min ischemia followed by $24-\mathrm{h}$ reperfusion

\begin{tabular}{|c|c|c|c|c|}
\hline Pathology & & $|R|$ & ATV & Necrotic area \\
\hline \multirow[t]{2}{*}{ Lean } & & + & - & $21.9 \pm 11.5$ \\
\hline & & + & + & $4.31 \pm 5.93^{*}$ \\
\hline \multirow[t]{4}{*}{ Simple steatosis } & A & + & - & $35.6 \pm 17.6$ \\
\hline & & + & + & $2.94 \pm 4.04^{*}$ \\
\hline & B & + & - & $37.2 \pm 27.7$ \\
\hline & & + & + & $1.75 \pm 2.21^{*}$ \\
\hline \multirow[t]{2}{*}{ NASH } & & + & - & $65.5 \pm 5.74$ \\
\hline & & + & + & $8.79 \pm 6.82^{*}$ \\
\hline
\end{tabular}

${ }^{*} P<0.05$ compared to untreated controls in the same liver histology group. Values are expressed as the mean \pm standard deviation $(n=6$ mice/group). Necrotic area is expressed as the percentage of total area of liver section at low magnification $(\times 4)$. Simple steatosis was derived by $(A)$,feeding wild-type mice a high-fat diet for 8 weeks, or (B),feeding foz/foz mice normal chow for 8 weeks. NASH was produced by feeding foz/foz mice a high-fat diet for 8 weeks. ATV, atorvastatin (10-day treatment); IRI, ischemia-reperfusion injury; NASH, non-alcoholic steatohepatitis.
ALT and the hepatic necrotic area at 24-h post-ischemic reperfusion, the protective efficacy of this single intravenous dose of atorvastatin was virtually equivalent to that of 10 days of oral atorvastatin (Fig. 4a,b). Further, the single intravenous dose of atorvastatin replicated the effects of 10-day oral atorvastatin therapy on the suppression of TLR4 in all experimental groups (Fig. 4c,d), and significantly attenuated TNF- $\alpha$, IFN- $\gamma$, and IL-6 release, compared to vehicle-treated animals with NASH, SS, and lean livers (Table 3). Single low-dose statin administered $24 \mathrm{~h}$ before IRI also activated eNOS, at least in the diet-induced SS liver (Supplementary Fig. S2a-c).

\section{Discussion}

Strategies to protect the fatty liver from accentuated injury during surgery or transplantation are much needed to improve the outcomes of liver resections in obese patients and to extend the use of marginal donor livers. ${ }^{27}$ None have yet been adopted routinely, so there is an outstanding need for readily-available agents that are safe and approved for human use. ${ }^{27}$ Statins are known to protect the lean liver, as well as other organs from ischemic damage, and our work confirms a recent report ${ }^{17}$ that such effects also extend to protection of fatty livers against hepatic IRI. Thus, a 10-day oral course of atorvastatin at doses equivalent to those used in humans virtually abolished hepatic IRI in all livers, irrespective of fatty liver pathology, including NASH. Further, the efficacy of atorvastatin against hepatic IRI we observed in fatty livers and NASH did not require the lowering of serum cholesterol. Our highly novel observation that similar hepatoprotection was conferred by a single low-dose of atorvastatin administered intravenously $24 \mathrm{~h}$
Figure 2 Effects of atorvastatin (ATV) and ischemia-reperfusion injury (IRI) on endothelial nitric oxide synthase (eNOS) expression and activation. (a) eNOS mRNA by quantitative reverse transcription-polymerase chain reaction; results normalized to GAPDH. (b) Western blot analysis (representative blots) of eNOS in sham-operated (c) and IRI (d) mice pretreated with ATV. (d) Phosphorylated eNOS expression which reflects eNOS activation. ${ }^{*} P<0.05$ compared to genotype-matched IRI group. ${ }^{*} P<0.05$ compared to genotypematched sham. HFD, high-fat diet; NC, normal chow; SS, simple steatosis; WT, wild type.

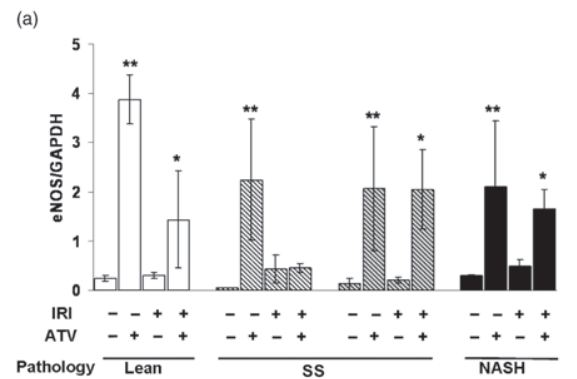

(c)
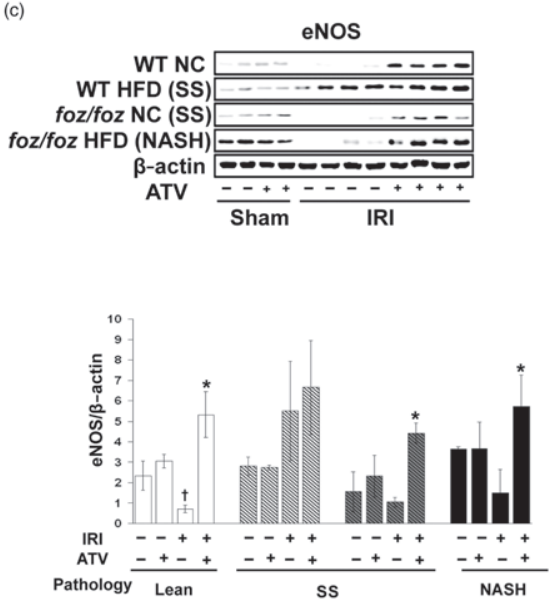

(b)

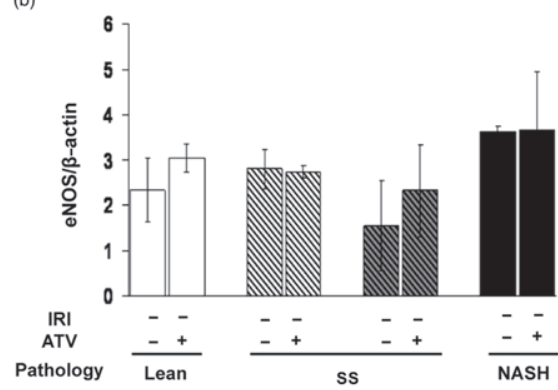

(d)

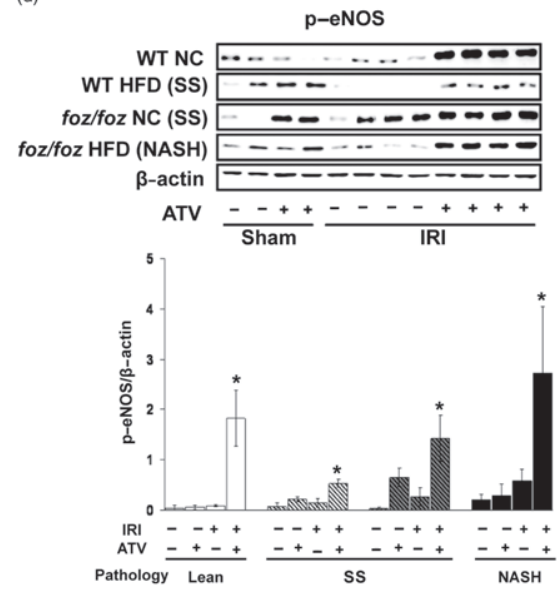


Table 2 Serum pro-inflammatory chemokines and cytokines following hepatic ischemia-reperfusion in naïve and 10-day atorvastatin-treated, lean, fatty, and NASH livers

\begin{tabular}{|c|c|c|c|c|c|c|c|c|c|}
\hline Pathology & & $|R|$ & ATV & MIP-1a (pg/mL) & MCP-1 (pg/mL) & TNF- $\alpha(p g / m L)$ & IL-12 p40 (pg/mL) & $\mathrm{IFN}-\gamma(\mathrm{pg} / \mathrm{mL})$ & IL-6 (pg/mL) \\
\hline \multirow[t]{3}{*}{ Lean } & & - & - & $107 \pm 0$ & $57.8 \pm 12.1$ & $111 \pm 31.1$ & $21 \pm 1.9$ & $22.3 \pm 3.17$ & $6.0 \pm 2.5$ \\
\hline & & + & - & $357 \pm 116$ & $260 \pm 27.3$ & $257 \pm 94.2$ & $157 \pm 6.78$ & $48.6 \pm 21.3$ & $84 \pm 33$ \\
\hline & & + & + & $273 \pm 94.2$ & $228 \pm 17.7$ & $233 \pm 57.7$ & $101 \pm 41.3$ & $37.3 \pm 6.67$ & $37 \pm 20^{*}$ \\
\hline \multirow[t]{4}{*}{ Simple steatosis } & A & + & - & $338 \pm 154$ & $301 \pm 56.9$ & $199 \pm 75.1$ & $107 \pm 16.2$ & $40.6 \pm 18.2$ & $49 \pm 11$ \\
\hline & & + & + & $489 \pm 78.2$ & $356 \pm 39.1$ & $218 \pm 85.7$ & $73 \pm 6.9^{*}$ & $79.1 \pm 10.8$ & $37 \pm 3.7^{*}$ \\
\hline & $B$ & + & - & $481 \pm 41.1$ & $1301 \pm 822.0$ & $311 \pm 127$ & $257 \pm 172$ & $49.3 \pm 18.4$ & $790 \pm 656$ \\
\hline & & + & + & $211 \pm 45.7^{*}$ & $118 \pm 48.6^{*}$ & $125 \pm 64.1^{*}$ & $93 \pm 6.9^{*}$ & $24.3 \pm 4.94^{*}$ & $22 \pm 13^{*}$ \\
\hline \multirow[t]{2}{*}{ NASH } & & + & - & $424 \pm 93.9$ & $585 \pm 144$ & $295 \pm 79.9$ & $163 \pm 20.5$ & $41.1 \pm 5.77$ & $285 \pm 187$ \\
\hline & & + & + & $270 \pm 36.2^{*}$ & $251 \pm 131^{*}$ & $177 \pm 31.9 *$ & $89.8 \pm 25.6^{*}$ & $27.7 \pm 4.87^{*}$ & $31 \pm 10 *$ \\
\hline
\end{tabular}

${ }^{*} P<0.05$ for comparison to both sham and respective untreated controls. Values are expressed as mean \pm standard deviation ( $n=4$ mice/group). Simple steatosis was produced by (A) feeding wild-type mice a high-fat diet for 8 weeks, or (B), feeding foz/foz mice normal chow for 8 weeks. NASH was produced by feeding foz/foz mice a high-fat diet for 8 weeks. ATV, atorvastatin (10-day treatment); IFN- $\gamma$, $\gamma$-interferon; IL, interleukin; IRI, ischemia-reperfusion injury; MCP-1, monocyte chemotactic protein-1; MIP-1a, macrophage inflammatory protein-1a; NASH, non-alcoholic steatohepatitis; TNF- $\alpha$, tumor necrosis factor- $\alpha$.

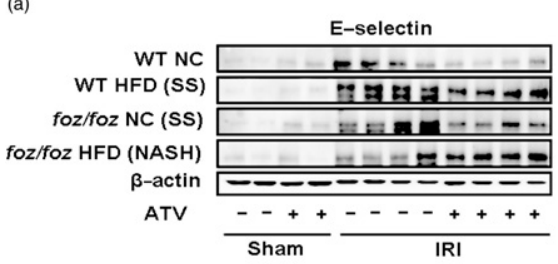

(c)



(e)

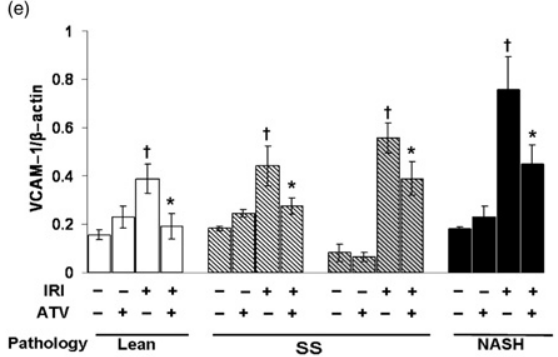

(b)

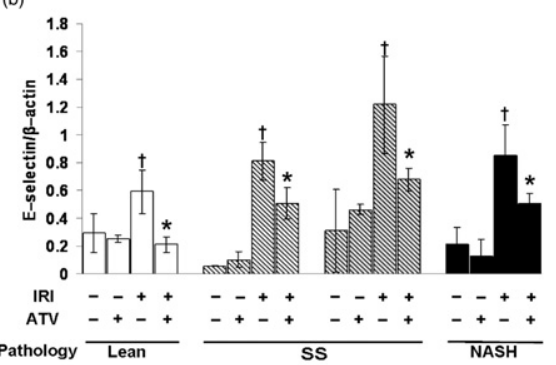

(d)

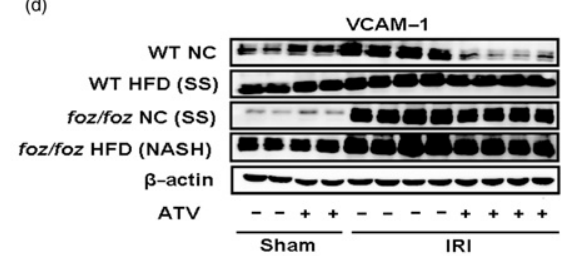

(f)



Figure 3 Effects of atorvastatin (ATV) and ischemia-reperfusion injury (IRI) on E-selectin, VCAM-1 expression, and serum thromboxane B2 (TxB2) levels. (a) E-selectin Western blots, (b) semiquantification analyses, (c) Vascular endothelial-cadherin Western blots and $(d, e)$ vascular cell adhesion molecule-1 (VCAM-1) analyses in hepatic lysates from lean, steatotic (high-fat diet [HFD] wild-type [WT] mice) and non-alcoholic steatohepatitis (NASH) livers. (f) ATV treatment for 10 days significantly reduced post-ischemic serum TxB2 levels in lean and steatotic livers, with a nonsignificant similar trend in $\mathrm{NASH} .{ }^{*} P<0.05$ compared to genotype-matched control with IRI. NC, normal chow; SS, simple steatosis. before the ischemic insult confirms that the molecular mechanisms, whereby statins protect both fatty and lean livers against hepatic IRI, extend beyond cholesterol-lowering effects on hepatocytes. Further, they indicate a more relevant clinical application of statins to protect fatty livers against hepatic IRI. In the present study, we used genetic and dietary models of SS, which we have shown do not exhibit increased hepatic cholesterol stores; this reflects lipidomic studies of human NAFLD, in which cholesterol retention occurs only with NASH. ${ }^{18}$
First, the protective effects we observed were best accounted for by the abrogation of post-ischemic TLR4 expression. TLR expressed on hepatocytes, Kupffer cells, and neutrophils play an important role in activating immune cells in IRI $^{28,29}$ via the MyD88-dependent, as well as MyD88-independent, activation of $\mathrm{NF}-\kappa \mathrm{B} ;{ }^{30-32}$ they cause the release of pro-inflammatory chemokines and cytokines (MIP-1a, MCP-1, TNF- $\alpha$, IL-1 $\beta$, IL-6, IL-12 p40, IL-18). ${ }^{30,31}$ In the present experiments, the IRI-mediated induction of TLR4 was approximately fourfold greater in livers 
Figure 4 Effects of single-dose $(5 \mathrm{mg} / \mathrm{kg})$, 24-h intravenous atorvastatin (ATV) on (a) serum alanine aminotransferase (ALT) levels, (b) liver necrotic areas, and (c,d) hepatic Tolllike receptor-4 (TLR4) expression after 24-h post-ischemic reperfusion in lean, simple steatotic (SS), and NASH livers. ATV was dissolved in DMSO and administrated intravenously. Data are expressed as mean \pm standard deviation ( $n=6$ mice/ group). ${ }^{*} P<0.05$ compared to genotypematched ischemia-reperfusion injury (IRI) group without statin. HFD, high-fat diet; NC, normal chow; WT, wild type. (a)

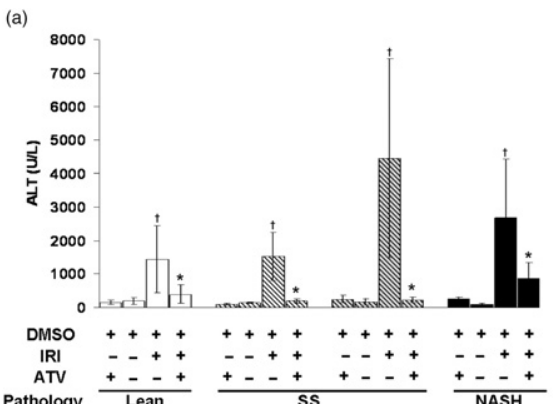

(c)

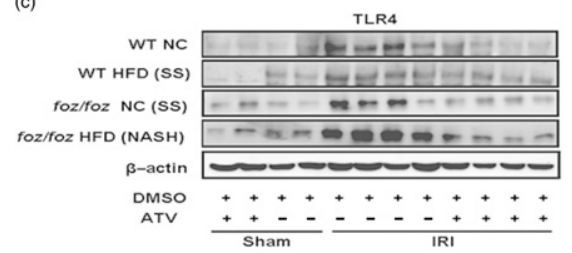

(b)

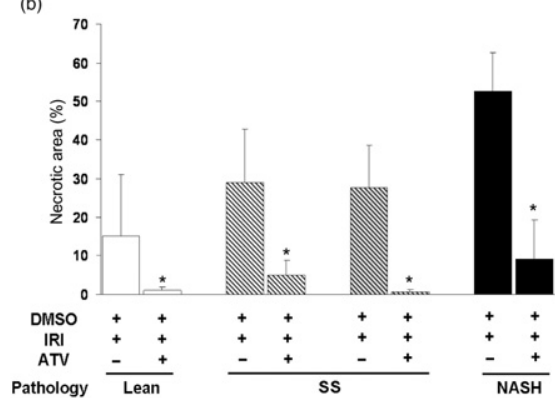

(d)

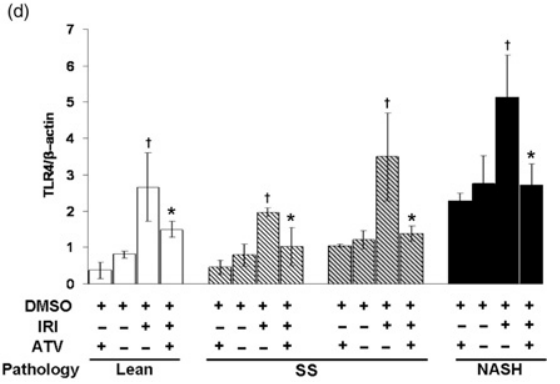

Table 3 Serum pro-inflammatory cytokine release following hepatic ischemia-reperfusion in naïve and 24-h atorvastatin-treated, lean, fatty, and NASH livers

\begin{tabular}{|c|c|c|c|c|c|c|}
\hline Pathology & & $\mid \mathrm{RI}$ & ATV & TNF- $\alpha(p g / m L)$ & IFN- $\gamma(\mathrm{pg} / \mathrm{mL})$ & IL-6 (pg/mL) \\
\hline \multirow[t]{4}{*}{ Lean } & & - & - & $0 \pm 0$ & $6.87 \pm 1.82$ & $192 \pm 21.2$ \\
\hline & & - & + & $0 \pm 0$ & $0 \pm 0$ & $31.8 \pm 29.2$ \\
\hline & & + & - & $527 \pm 49.5$ & $166 \pm 24.5$ & $515 \pm 63.6$ \\
\hline & & + & + & $137 \pm 25.2^{*}$ & $35.3 \pm 12.7^{*}$ & $297 \pm 72.5^{*}$ \\
\hline \multirow[t]{8}{*}{ Simple steatosis } & A & - & - & $0 \pm 0$ & $8.72 \pm 6.82$ & $105 \pm 49.2$ \\
\hline & & - & + & $8.88 \pm 0.62$ & $0 \pm 0$ & $88.5 \pm 31.2$ \\
\hline & & + & - & $725 \pm 100$ & $183 \pm 40.6$ & $700 \pm 237$ \\
\hline & & + & + & $179 \pm 46.4^{*}$ & $29.4 \pm 19.3^{*}$ & $267 \pm 119 *$ \\
\hline & B & - & - & $0 \pm 0$ & $8.75 \pm 4.88$ & $134 \pm 15.1$ \\
\hline & & - & + & $0 \pm 0$ & $0 \pm 0$ & $138 \pm 34.6$ \\
\hline & & + & - & $742 \pm 94$ & $451 \pm 14.8$ & $1502 \pm 431.2$ \\
\hline & & + & + & $85.8 \pm 19.4^{*}$ & $52.1 \pm 20.1^{*}$ & $303 \pm 105^{*}$ \\
\hline \multirow[t]{4}{*}{$\mathrm{NASH}$} & & - & - & $73.7 \pm 28.9$ & $46.8 \pm 2.66$ & $125 \pm 13.4$ \\
\hline & & - & + & $0 \pm 0$ & $0 \pm 0$ & $57.5 \pm 34.6$ \\
\hline & & + & - & $565 \pm 179$ & $415 \pm 35.9$ & $835 \pm 57.7$ \\
\hline & & + & + & $256 \pm 59.9^{*}$ & $152 \pm 19.4^{*}$ & $331 \pm 75.7^{*}$ \\
\hline
\end{tabular}

${ }^{*} P<0.05$ for comparison of both sham and respective untreated controls. Values are expressed as mean \pm standard deviation ( $n=4 /$ per group). Simple steatosis was produced by (A) feeding wild-type mice a high-fat diet for 8 weeks, or (B) feeding foz/foz mice normal chow for 8 weeks. NASH was produced by feeding foz/foz mice HF diet for 8 weeks. ATV, atorvastatin (10-day treatment); IFN- $\gamma$, $\gamma$-interferon; IL-6, interleukin-6; IRI, ischemia-reperfusion injury; NASH, non-alcoholic steatohepatitis; TNF- $\alpha$, tumor necrosis factor- $\alpha$.

showing NASH, and twofold higher in SS than in lean livers, as was the corresponding activation of NF-KB. Increased TLR4 expression is a plausible explanation for the greater susceptibility of fatty livers to IRI, because basal TLR4 expression is increased in fatty livers, particularly those showing NASH (Fig. 1c). The latter finding argues against the alternative explanation that the enhanced expression of TLR4 after IRI in fatty livers could be secondary to more severe post-ischemic liver injury, rather than playing a causative role; further evidence that TLR4 signaling to NF- $\mathrm{KB}$ via MyD88 is mechanistically relevant to IRI is that $\mathrm{TLR}^{-/-}$and $\mathrm{MyD}^{-/-}$mice are refractory to $\mathrm{IRI}^{31,32}$ (Supplementary Fig. S3).

Second, the implication of the statin-induced abrogation of a TLR4 response to IRI in fatty liver disease is via its "downstream" effect of preventing NF- $\kappa B$ activation. The present results clearly indicate that degradation of I $\mathrm{KB}-\alpha$, the cytosolic inhibitor of $\mathrm{NF}-\kappa \mathrm{B}$, after IRI was prevented by statin pretreatment, as was the resultant nuclear expression of p65. With atorvastatin treatment before IRI, the abrogation of NF- $\mathrm{KB}$ activation prevented elaboration of the pro-inflammatory Th1 cytokines, TNF- $\alpha$, IL-12 p40, 
and IFN- $\gamma$, and also decreased IL- 6 production after IRI. Because much evidence supports a role for IL- 6 mediating the hepatoprotective and pro-proliferative effects of preconditioning, ${ }^{2,6,27,33,34}$ a third point to note is that, in addition to the clear hepatoprotective efficacy of atorvastatin, there was no deleterious effect on hepatocyte proliferative pathways, such as $\mathrm{p} 38$ stress-activated protein kinase and cyclin D1; instead, these pathways were more activated after IRI in the livers of statin-treated mice (not shown).

Fourth, statin pretreatment reduced secretion of both macrophage (MIP-1a, MCP-1) and PMN (IL-6) chemokines. The importance of such favorable modulation of these pro-inflammatory molecules was reflected by the impressive reduction in liver inflammation by atorvastatin, in association with a $\geq 85 \%$ reduction in the necrotic area in NASH or steatotic livers. Together with the macrophage and PMN chemokines, we also found that the expression of pro-inflammatory adhesion molecules after IRI was abolished by atorvastatin. The adhesion molecules most favorably altered by statin treatment were those expressed on hepatic endothelial cells (E-selectin, VCAM-1), again arguing against the proposed primary effect of statins on hepatocyte mitochondria. ${ }^{17}$ Finally, 10 days of atorvastatin therapy also decreased TxB2 production after IRI. Thromboxanes are suppressed by eNOS, and their suppression would conceivably contribute to the preservation of microvascular blood flow after IRI, as shown with Diannexin. ${ }^{5}$

A fifth important finding pertinent to sinusoidal blood flow was that atorvastatin treatment had profound and isoform-specific effects on pathways of hepatic NO production. Ten days of oral atorvastatin increased hepatic eNOS mRNA and protein approximately 10-fold, irrespective of underlying liver pathology, and this induction was maintained after IRI. More importantly, the activation of enzymically-active eNOS (Fig. 2c,d) after IRI was consistently and impressively augmented by prior atorvastatin treatment. Thus, in response to IRI, clear differences in eNOS and p-eNOS expressions are observed. The increase in total eNOS in response to atorvastatin reflects the efficacy of statins to modulate NO production at the transcriptional level. ${ }^{35-39} \mathrm{We}$ also found that ATV also suppressed inducible NOS (iNOS) mRNA induction (not shown), which is under NF- $\mathrm{KB}$ regulatory control. The combined effect of iNOS suppression and eNOS induction/activation would be to diminish intracellular NO accumulation, with its unwanted effects on reactive nitrogen species generation and mitochondrial injury, at the same time promoting the endothelial cell secretion of NO. Only the latter has beneficial effects by causing vasodilatation of the hepatic microvasculature, opposing apoptosis of SEC, and suppressing thromboxane production. ${ }^{5,6}$ Interestingly, while we observe little change in eNOS protein expression in livers from sham-operated, atorvastatin-treated mice, we acknowledge that this is in contradiction to other studies, where increments in eNOS are noted in myocardium and human umbilical vascular endothelial cells. ${ }^{35-39}$ Thus, it is possible that statins de novo in liverderived cells have a different effect.

For patients scheduled for major liver resections, and for livingrelated donors, the usual approach to liver transplantation in Asia (the usual approach to liver transplantation in Asia), a 10-day oral treatment course with a statin would be feasible to prevent hepatic IRI. However, for urgent surgery, as with cadaveric liver transplantation, such oral therapy is not feasible. Instead, a single intravenous injection of atorvastatin $24 \mathrm{~h}$ before the hepatic ischemic insult, as shown here to be similarly effective as the longer oral regimen, could be utilized. Further studies are required to clarify whether the anti-inflammatory effects of statins explain all of its hepatoprotective effects against liver IRI, or whether endothelial cell protection and eNOS-mediated NO production, with its beneficial effects on cytoprotection and microcirculatory blood flow, are also important. Meanwhile, the results of the present and another recent study ${ }^{17}$ are so compelling that clinical trials of statin therapy to protect fatty livers against hepatic IRI and preservation injury would be of immense interest.

\section{Acknowledgment}

This research was supported by project grant no. 1010082 of the Australian National Health and Medical Research Council (NHMRC).

\section{References}

1 Farrell GC, Teoh NC, McCuskey RS. Hepatic microcirculation in fatty liver disease. Anat. Rec. (Hoboken) 2008; 291: 684-92.

2 Bahde R, Spiegel HU. Hepatic ischaemia-reperfusion injury from bench to bedside. Br. J. Surg. 2010; 97: 1461-75.

3 Jaeschke H. Reactive oxygen and mechanisms of inflammatory liver injury: present concepts. J. Gastroenterol. Hepatol. 2011; 26 (Suppl. 1): 173-9.

4 Jaeschke H, Lemasters JJ. Apoptosis versus oncotic necrosis in hepatic ischemia/reperfusion injury. Gastroenterology 2003; 125: 1246-57.

5 Teoh NC, Ito Y, Field J et al. Diannexin, a novel annexin V homodimer, provides prolonged protection against hepatic ischemiareperfusion injury in mice. Gastroenterology 2007; 133: 632-46.

6 Teoh NC, Farrell GC. Hepatic ischemia reperfusion injury: pathogenic mechanisms and basis for hepatoprotection. J. Gastroenterol. Hepatol. 2003; 18: 891-902.

7 Temkin V, Karin M. From death receptor to reactive oxygen species and c-Jun N-terminal protein kinase: the receptor-interacting protein 1 odyssey. Immunol. Rev. 2007; 220: 8-21.

8 Li Q, Verma IM. NF-kappaB regulation in the immune system. Nat. Rev. Immunol. 2002; 2: 725-34.

9 Rivera CA, Adegboyega P, van Rooijen N, Tagalicud A, Allman M, Wallace M. Toll-like receptor-4 signaling and Kupffer cells play pivotal roles in the pathogenesis of non-alcoholic steatohepatitis. J. Hepatol. 2007; 47: 571-9.

10 Csak T, Velayudham A, Hritz I et al. Deficiency in myeloid differentiation factor- 2 and Toll-like receptor 4 expression attenuates non-alcoholic steatohepatitis and fibrosis in mice. Am. J. Physiol. Gastrointest. Liver Physiol. 2011; 300: G433-41.

11 Teoh NC, Williams J, Hartley J, Yu J, McCuskey RS, Farrell GC. Short-term therapy with peroxisome proliferation-activator receptor-alpha agonist $\mathrm{Wy}-14,643$ protects murine fatty liver against ischemia-reperfusion injury. Hepatology 2010; 51: 996-1006.

12 Atar S, Ye Y, Lin Y et al. Atorvastatin-induced cardioprotection is mediated by increasing inducible nitric oxide synthase and consequent S-nitrosylation of cyclooxygenase-2. Am. J. Physiol. Heart Circ. Physiol. 2006; 290: H1960-8.

13 Dibazar F, Hajipour B, Hosseinian MM, Hemmati MR, Ghandiha A Simvastatin decreases hepatic ischaemia/reperfusion-induced liver and lung injury in rats. Folia Morphol. (Warsz) 2008; 67: 231-5.

14 Lai IR, Chang KJ, Tsai HW, Chen CF. Pharmacological preconditioning with simvastatin protects liver from ischemia-reperfusion injury by heme oxygenase-1 induction. Transplantation 2008; 85: 732-8. 
15 Liao JK, Laufs U. Pleiotropic effects of statins. Annu. Rev. Pharmacol. Toxicol. 2005; 45: 89-118.

16 Rikitake Y, Liao JK. Rho GTPases, statins, and nitric oxide. Circ. Res. 2005; 97: 1232-5.

17 Llacuna L, Fernandez A, Von Montfort C et al. Targeting cholesterol at different levels in the mevalonate pathway protects fatty liver against ischemia-reperfusion injury. J. Hepatol. 2011; 54: 1002-10.

18 Puri P, Baillie RA, Wiest MM et al. A lipidomic analysis of non-alcoholic fatty liver disease. Hepatology 2009; 46: 1081-90.

19 Arsov T, Larter CZ, Nolan CJ et al. Adaptive failure to high-fat diet characterizes steatohepatitis in Alms1 mutant mice. Biochem. Biophys. Res. Commun. 2006; 342: 1152-9.

20 Larter CZ, Yeh MM. Animal models of NASH: getting both pathology and metabolic context right. J. Gastroenterol. Hepatol. 2008; 23: 1635-48.

21 Larter CZ, Yeh MM, Van Rooyen DM et al. Roles of adipose restriction and metabolic factors in progression of steatosis to steatohepatitis in obese, diabetic mice. J. Gastroenterol. Hepatol. 2009; 24: 1658-68.

22 Van Rooyen DM, Larter CZ, Haigh WG et al. hepatic free cholesterol accumulates in obese, diabetic mice and causes non-alcoholic steatohepatitis. Gastroenterology 2011; 141: 1393-403.

23 Uchida Y, Ke B, Freitas MC et al. T-cell immunoglobulin mucin-3 determines severity of liver ischemia/reperfusion injury in mice in a TLR4-dependent manner. Gastroenterology 2010; 139: 2195-206.

24 Ellett JD, Evans ZP, Atkinson C, Schmidt MG, Schnellmann RG, Chavin KD. Toll-like receptor 4 is a key mediator of murine steatotic liver warm ischemia/reperfusion injury. Liver Transpl. 2009; 15: 1101-9.

25 Arumugam TV, Okun E, Tang SC, Thundyil J, Taylor SM, Woodruff TM. Toll-like receptors in ischemia-reperfusion injury. Shock 2009; 32: 4-16.

26 Stalker TJ, Lefer AM, Scalia R. A new HMG-CoA reductase inhibitor, rosuvastatin, exerts anti-inflammatory effects on the microvascular endothelium: the role of mevalonic acid. $B r . J$. Pharmacol. 2001; 133: 406-12.

27 de Rougemont O, Lehmann K, Clavien PA. Preconditioning, organ preservation, and postconditioning to prevent ischemia-reperfusion injury to the liver. Liver Transpl. 2009; 15: 1172-82.

28 Zhai Y, Shen XD, O'Connell R et al. Cutting edge: TLR4 activation mediates liver ischemia-reperfusion inflammatory response via IFN regulatory factor 3-dependent MyD88-independent pathway. J. Immunol. 2005; 173: 7115-19.

29 Mencin A, Kluwe J, Schwabe RF. Toll-like receptors as targets in chronic liver diseases. Gut 2009; 58: 704-20.

30 Teoh N, Field J, Farrell G. Interleukin-6 is a key mediator of the hepatoprotective and pro-proliferative effects of ischaemic preconditioning in mice. J. Hepatol. 2006; 45: 20-7.

31 Teoh NC. Hepatic ischemia reperfusion injury: contemporary perspectives on pathogenic mechanisms and basis for hepatoprotection-the good, bad and deadly. J. Gastroenterol. Hepatol. 2011; 26: 1807-16.

32 Zhai Y, Shen XD, O'Connell R et al. Cutting edge: TLR4 activation mediates liver ischemia-reperfusion inflammatory response via IFN regulatory factor 3-dependent MyD88-independent pathway. J. Immunol. 2004; 173: 7115-19.

33 Teoh N, Dela Pena A, Farrell G. Hepatic ischemic preconditioning in mice is associated with activation of NF-kappaB, p38 kinase, and cell cycle entry. Hepatology 2002; 36: 94-102.

34 Kannerup AS, Grønbaek H, Funch-Jensen P, Tønnesen E, Jørgensen RL, Mortensen FV. Cytokine changes during warm ischemia and reperfusion of the pig liver with or without preconditioning. Eur. Surg. Res. 2009; 42: 216-22.
35 Urbich C, Dimmeler S. Risk factor for coronary artery disease, circulating endothelial progenitor cells, and the role of HMG-Coa reductase inhibitors. Kidney Int. 2005; 67: 1672-6.

36 Wolfrum S, Grimm M, Heidbreder M et al. Acute reduction of myocardial infarct size by a hydroxymethyl glutaryl coenzyme A reductase inhibitor is mediated by endothelial nitric oxide.

J. Cardiovasc. Pharmacol. 2003; 41: 474-80.

37 Parmar KM, Nambudin V, Dai G, Larman HB, Gimbrone MA Jr, Garcia-Cardena G. Statins exert endothelial artheroprotective effects via the KLF2 transcription factor. J. Biol. Chem. 2005; 280: 26714-19.

38 Prakash P, Khanna V, Singh V et al. Atorvastatin protects against ischemia-reperfusion injury in fructose-induced insulin resistant rats. Cardiovasc. Drugs Ther. 2011; 25: 285-97.

39 Sen-Banerjee S, Mir S, Lin Z et al. Kruppel-like factor 2 as a novel mediator of statin effects in endothelial cells. Circulation 2005; 112: 720-6.

\section{Supporting information}

Additional Supporting Information may be found in the online version of this article:

Figure S1 Atorvastatin (ATV) dampens hepatic polymorphonuclear leukocyte (PMN) and macrophage infiltration after ischemia-reperfusion injury (IRI). (a) Representative immunohistochemical images for of PMN infiltration shown by myeloperoxidase (MPO) staining at $24 \mathrm{~h}$ after hepatic IRI. Livers showed strong MPO immunostaining (arrows) after IRI, irrespective of whether they were (a) lean or (b) steatotic (high-fat diet wild-type mice), or (c) showed non-alcoholic steatohepatitis (NASH) $(\times 400$ magnification). Atorvastatin treatment markedly reduced PMN infiltration (d-f). (b) Quantification of F4/80-positive cells (macrophages) after hepatic IRI. IRI induced hepatic macrophage recruitment in lean, simple steatosis (SS), and NASH livers; atorvastatin ameliorated macrophage immunoreactivity, particularly in livers with SS and NASH. $* P \leq 0.05$ compared to matched control with IRI. HPF, high power field ( $\times 400$ magnification).

Figure S2 Effects of single-dose ( $5 \mathrm{mg} / \mathrm{kg}), 24-\mathrm{h}$ intravenous atorvastatin on endothelial nitric oxide synthase (eNOS) and phosphorylated eNOS (p-eNOS) expression after 24-h post-ischemic reperfusion in lean, simple steatosis, and non-alcoholic steatohepatitis livers. Data are expressed as mean \pm standard deviation ( $n=6$ mice per group). (a) Western blot of eNOS and (b) p-eNOS in hepatic lysates, and (c) ratio of p-eNOS to eNOS, which reflects active eNOS. $* P<0.05$ compared to respective genotype-matched group with ischemia-reperfusion injury.

Figure S3 MyD88 ${ }^{-1-}$ mice are refractory to liver ischemiareperfusion injury. Hepatocellular injury, as measured by serum levels of alanine aminotransferase (ALT) in MyD88 gene-deleted mice subjected to $60 \mathrm{~min}$ of ischemia and $24 \mathrm{~h}$ of reperfusion.

Table S1 Serum cholesterol levels in naïve and 10-day atorvastatin-treated lean, fatty and non-alcoholic steatohepatitis livers subjected to ischemia-reperfusion injury (IRI).

Please note: Wiley-Blackwell are not responsible for the content or functionality of any supporting materials supplied by the authors. Any queries (other than missing material) should be directed to the corresponding author for the article. 